\title{
Language typology and statistics
}

Emil O. W. Kirkegaard

\section{Introduction}

Typology is a subfield of linguistics concerned with the classification of languages and the finding of patterns among the different classifications, or to put it another way, it is the study of linguistic diversity (Daniel, 2011; Moravcsik, 2013; Ramat, 2011; Velupillai, 2012). Hundreds if not thousands of such patterns has been proposed in the literature going back many decades (Greenberg, 1966), if not centuries (Ramat, 2011).

Apparently typology has not traditionally been done using standard statistical methods (Daniel, 2011). This may seem odd to the (non-linguist) quantitative scientist because the task of trying to infer properties of a population of languages from samples of languages is a quintessential statistical task.

The purpose of this paper is to argue that typology faces problems that can best be overcome with an explicit statistical approach. This is done by analyzing typological data from the widely used World Atlas of Language Structures (WALS) database using statistical methods.

\subsection{Language sampling and the goal of typology}

Generally, when typologists try to find language patterns (see Section 1.3), they rely on large collections (datasets) of information about languages built by other linguists ("armchair typology", (Daniel, 2011)). This is because it would be an insurmountable amount of work to have to learn hundreds of languages in order to classify them according to various features oneself (Velupillai, 2012). There are some very large datasets of language features (see Section 2), yet they cover only a very small fraction of languages that have ever existed. According to one estimate (Moravcsik, 2013, p. 17), the number of (human) languages that has existed or still exists is about 240,000. Of these, only 3\% are still alive (and 50-90\% are expected to go extinct before 2100 (Grenoble, 2011)), and of the ones that are still alive, we have descriptions (of varying quality and detail) of about 33\%. Assuming that we generally do not have descriptions of extinct languages (true in most cases), this means that we have descriptions of about 2300 languages, or about 1\% of those that have existed so far.

\subsubsection{What is the language population of interest for typology?}

As described by multiple authors, the goal is typology is to find differences between languages and patterns among the differences, but which languages? In statistics, the set of units that we are interested in is called the population. Unfortunately, it is almost never possible to collect data about the entire population, so one must instead rely on data from subsets, or samples from the population. Perhaps most statistical theory, called inferential statistics (Cox, 2006), is concerned with the question of how we can infer properties of the population while only having access to samples.

However, before one should consider methods, one should consider the goal. The answer to the 
question of which population is the one we are interested in is not so obvious as one might think. For instance, if the population of interest is that of all languages, we immediately face a problem: almost all our data concern languages that exist now or recently. To be able to conclude something about all languages, one must make the assumption that this population is invariant over time, at least with respect to the language feature(s) of interest (Daniel, 2011; Maslova, 2000). It is possible to study languages of the past to see if there seems to be a change in the distribution of the feature(s) over time (language evolution), but we are still left in the dark about possible future changes in the feature(s) (Velupillai, 2012).

In light of this problem, one could give up the goal of trying to estimate of the properties of the population of all languages and instead settle for the easier task of estimating the properties of the population of languages that exist or have existed the last couple of thousand years, i.e. those we have data for. This makes it statistically more justifiable to make inferences about the population of interest, but also restricts the conclusions that can be drawn from the inferred patterns. When typologists find reasonably strong patterns, they often offer causal explanations for why we observe the patterns we do. These explanations may be cognitive (cognitive foundations of human language ability á la generative grammar or processing requirements) or functional (this feature tends to evolve because it makes communication easier) (Daniel, 2011; Moravcsik, 2013, Chapter 7). To reach conclusions of this sort, it would seem that one needs information about the population of all languages, not just the recent ones. Although perhaps one could make the counterargument that one is interested in only the recent cognitive foundations of human language ability or the recent language selection pressures.

\subsubsection{Representativeness}

Supposing that the above problems are solved to satisfaction, there is the question of the representativeness of language samples. Typologists often take great care to select a representative subset of languages so as to avoid non-typological influences: shared language history, language contact and shared cultural environment (Moravcsik, 2013, p. 5). In practice, this means that one would try to select a sample of languages that originates in a variety of parts of the world (in contrast to all of them originating in e.g. Africa), that belong to different language families (in contrast to all belonging to e.g. the indo-European family) and that did not share obvious cultural environments (in contrast to e.g. all being used in industrialized societies).

\subsubsection{Constructed languages}

Above I have talked about all languages, but perhaps most typologists would exclude the class of constructed languages (e.g. Esperanto, Klingon and the languages from the Tolkien universe) when making statements about proposed language universals (Libert, 2013b). For instance, one proposed pattern is:

1. If in a given language there is a separate term for 'leg' (as opposed to 'foot') then there is also a term for 'arm' (as opposed to 'hand'). (Moravcsik, 2013, p. 20; Brown, 1976)

I doubt that Brown would consider the pattern falsified if someone were to construct a language that had a word for leg but no word for arm (such as a language destined for creatures that have no arms but many legs (Okrent, 2009)). On the other hand, some proponents of generative grammar postulate that 
certain proposed patterns reflect a universal grammar (UG) (Daniel, 2011). Thus, if one could construct a language that humans can demonstrably use that violates one of the proposed UG-patterns, it is not so obvious what one should conclude about that. In general, however, constructed languages have generally not received much attention (Libert, 2013a, 2013b).

\subsection{Language features}

To classify languages one must first notice some feature of language where languages differ. A feature can be any part of languages including semantic, lexical, syntactic, morphological, phonological/phonetic patterns, orthographical and everything in between (Daniel, 2011; Moravcsik, 2013). Often what happens is that one only notices a feature when one becomes acquainted with two or more languages that differ on that feature (Velupillai, 2012, p. 16). For instance, to a native English speaker it may seem obvious that subjects always come before verbs that then come before objects (SVO-order). He might never consider uttering sentences in some other order except perhaps for humorous effect. Such a person might be surprised to read a few glossed sentences from Hawaiian, which has VSO order.

\subsection{Types of patterns}

Typology has developed its own terminology for describing the kinds of patterns that the researchers attempt to discover. The terminology is based on that from formal logic (e.g. LePore \& Cumming, 2009), but is not quite as extensive.

The weakest claim is the existential statement, namely that some pattern exists in at least one language. For instance:

2. At least one language has an oral stop.

This claim is obviously true since English has more than one. A stronger claim is the universal (also called the universal generalization). For instance:

3. All languages have an oral stop.

This is a much stronger claim; a single counter-example is enough to disprove it. A search in the The UCLA Phonological Segment Inventory Database (http://www.linguistics.ucla.edu/faciliti/sales/software.htm), which has data for 451 languages, does not reveal a language that has no oral stops. However, it is still possible that some language that was not included lacks an oral stop. Hence (in a Popperian fashion, (Chalmers, 1999; Sokal, 2008)), we cannot conclude that the generalization is true, merely that it has not been disproved so far.

\subsubsection{Statistical patterns}

In practice, universals like pattern 3 are rare because usually there are a few counterexamples to some proposed pattern. In this case, we might still want to make a note of the pattern even though it is not strictly true. Instead, we may thus talk about statistical or probabilistic patterns (Moravcsik, 2013, p. 14). For instance, if we tomorrow discover a new language that has no oral stops, we might want to give up pattern 3 and instead propose:

4. Almost all languages have oral stops. 
The quantifier almost falls in between the existential quantifier at least one and the universal quantifier all. One can vary the exact quantifier so that it fits with the data one has. For instance, if we examine a large dataset and find that $80 \%$ of languages have a given feature, we might want to use the quantifier most. If for another feature, we find that $98 \%$ of languages have it, we might opt for almost all.

\subsubsection{Conditional patterns}

Pattern 1 is different from all the other patterns we have seen so far because it is conditional; it says that if something is the case for a language, then something else is also the case. This is in contrast to pattern 3 which makes a statement about the entire set of languages. It is common to refer to the patterns like pattern 1 as conditional or restricted or implicational patterns and reversely to those like pattern 3 as unrestricted.

Confusingly, it is common to refer to patterns like pattern 4 as statistical universals (Moravcsik, 2011, 2013, p. 16; Velupillai, 2012, p. 31).

\subsection{Implicational universals as conditional probabilities}

What typologists call implicational universals is what is called conditional probabilities in statistics and other areas of science. They have the form:

The probability of A given B.

where A and B could be anything, such as features of language (Kruschke, 2011, p. 38). The distinction between absolute and statistical universals is merely whether the conditional probability is equal to 1 (100\%) or merely above 0.5 .

\section{Dataset}

A popular database of typological information is the The World Atlas of Language Structures (WALS) (Dryer \& Haspelmath, 2013). The version I downloaded for the purpose of doing this analysis contained data about 2679 different languages spanning 192 different features (dated to 2015-07-30). There are other datasets such as those offered by John Alderete on his website (http://anderei.net/datasets/) or those referenced on the website for the Association of Linguistic Typology (http://www.linguistic-typology.org/resources.html). However, I was not able to find any public dataset more comprehensive than WALS.

\subsection{Missing and 'missing' data}

There is a substantial amount of 'missing' data in the WALS dataset, in fact approximately 85\% of the cells are empty. This does not necessarily mean that the data is truly missing. Some features in the dataset only make sense given that a language has as given other feature. For instance, the feature $95 \mathrm{~A}$ Relationship between the Order of Object and Verb and the Order of Adposition and Noun Phrase only makes sense if the language has objects, verbs, adpositions and noun phrases. Other features are restricted to a subset a languages, e.g. 10B Nasal Vowels in West Africa (restricted to West African languages) or The Position of Negative Morphemes in SVO Languages (which cannot include data from languages that do not have SVO order) ${ }^{1}$.

1 There seems to be an error because it does include one OSV language. 
However, for many of the cells, presumably the reason there is no data is that it is unknown what the status of the feature is in that language (perhaps because the language is extinct) or that no one has yet added it to the dataset. See Velupillai (2012) for additional discussion of this dataset.

There are two simple ways one can examine the missing data. One can look at it from the perspective of languages (cases) and note how many features there is data about, or from the perspective of features (variables) and note how many languages there is data about. Figures 1 and 2 show distributions of missing data in the WALS dataset.

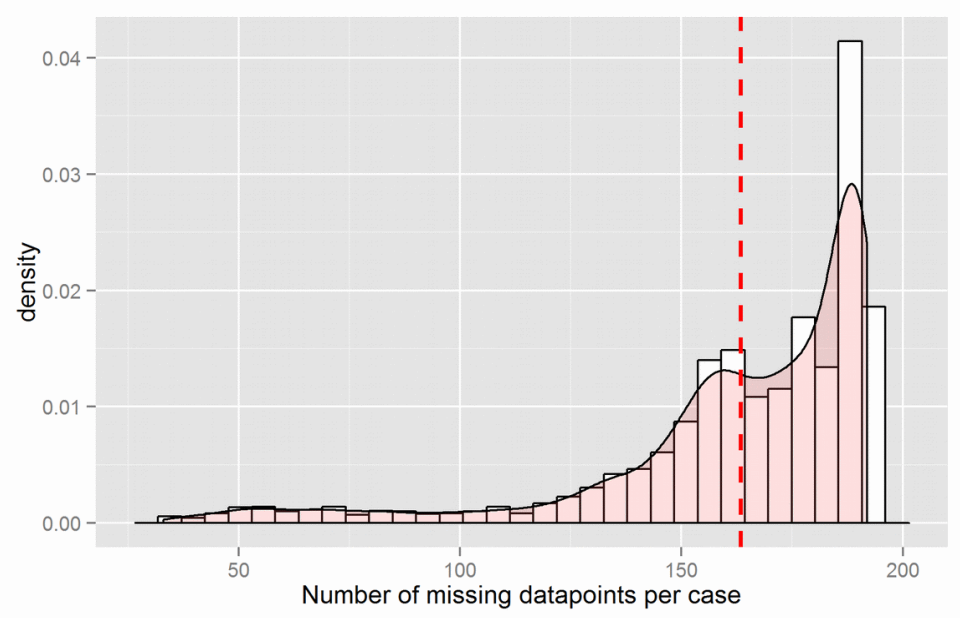

Figure 1: Distribution of missing data by language. The red line is the mean.

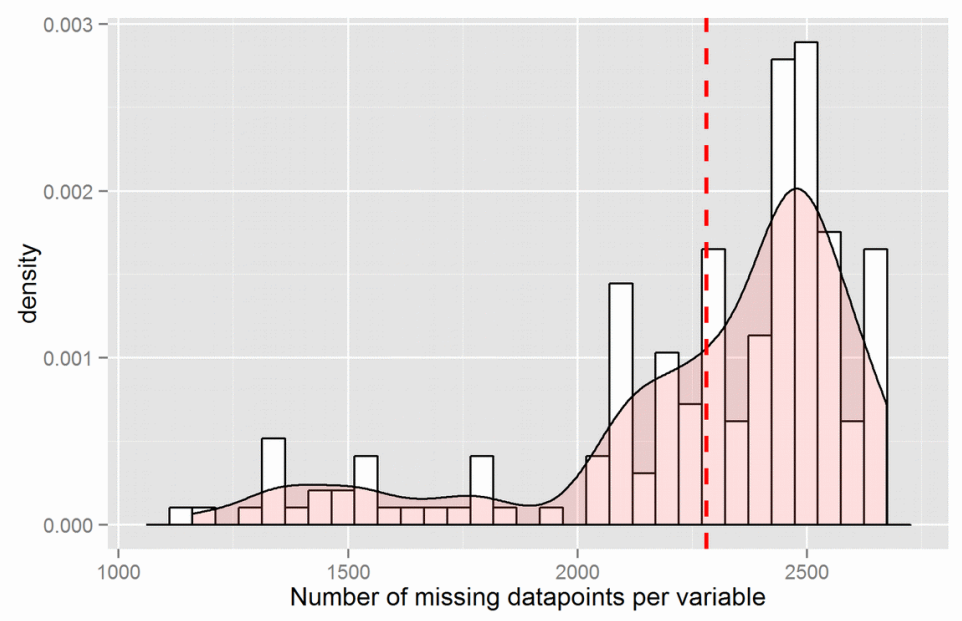

Figure 2: Distribution of missing data by feature. The red line is the mean.

As expected from the overall about of missing data (85\%), we see that most languages have 100s of missing datapoints with a mean of about 160 . Similarly, we see that most features have thousands of missing datapoints with a mean of about 2250.

It is important to be explicit about the missing data because many statistical methods will not work if there is missing data, while others will silently subset the data to a subset without missing data. This 
means that one must use a subset of the dataset, both in terms of features and languages for analysis. Results may strongly depend on which subset is chosen. To investigate whether there were regional differences in the proportion of missing data, I calculated the mean proportion of missing data by macroarea ('continents') as given by WALS. Figure 3 shows the results.

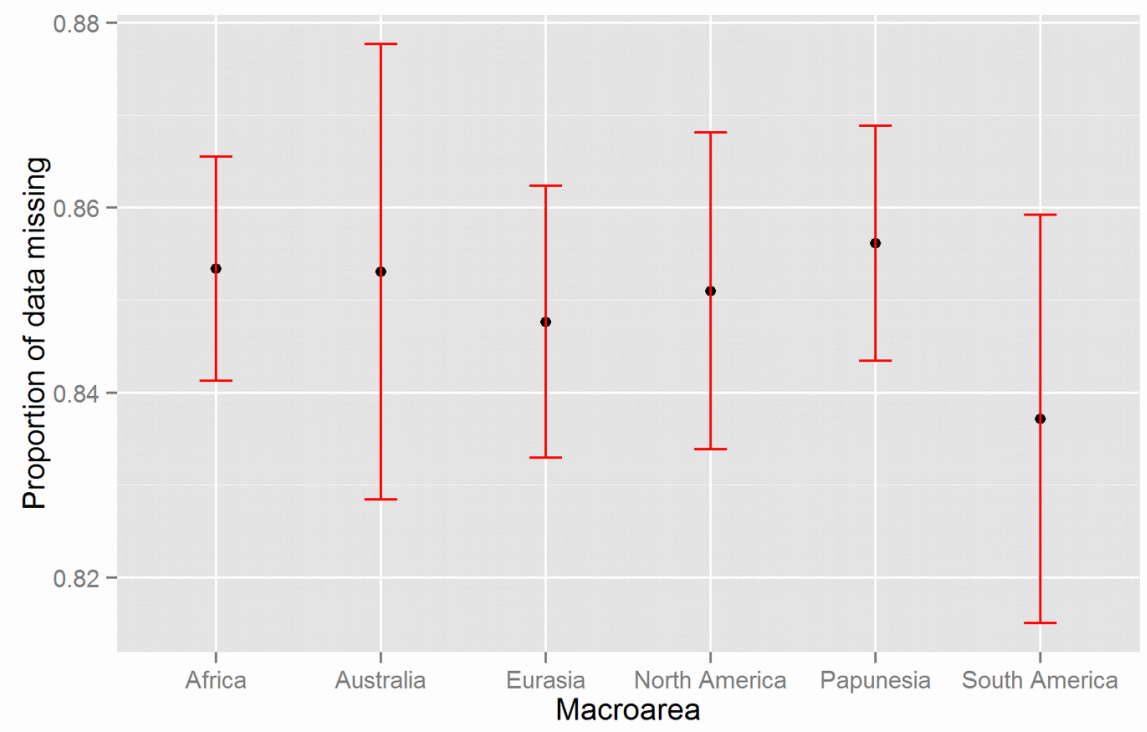

Figure 3: Proportion of missing data by macroarea.

Thus, we see that there do not seem to be stark differences in the proportion of missing data by continent, so sampling bias seems unlikely to result from that direction.

\section{The nature of the typological data}

\subsection{Measurement}

Measurement is central to science. Scientists choose some aspect of reality, find a way to measure it, and then do it. This is harder than it sounds because it is not easy to find or invent a good measure of some construct or phenomenon of interest. Take for instance temperature. Today we measure temperature using cheap standardized thermometers available for consumer use. However, it was not always easy to measure temperature and temperature itself is an abstract concept that was first later given a rigorous physio-chemical definition (the movement of small bodies of matter).

\subsubsection{Levels of measurement}

There multiple levels of measurement (Navarro, 2015, p. 14). Which level one's data is in constrains which statistical methods one can use.

\subsubsection{Nominal}

The lowest level of measurement is categorical (or nominal). This means that we can distinguish between multiple categories of units and we can name the categories, but we cannot rank the categories in any obvious way. An example of such data might be movie genres. We can assign names to these, but they don't have any order. 
With this level of measurement, one cannot use many mathematical operations. For instance, it makes no sense to try to calculate a mean of names. One can, however, count the number of units in each category.

\subsubsection{Ordinal}

The next level of data is ordinal, here we are able to order the units in terms of something. For instance, humans are able to feel the temperature of objects by touching them. However, this does not output a number of some kind and we only get a feeling of how warm or cold something is. Crucially, with ordinal data one cannot say anything about the relative distances (if that makes sense) between the units on the scale of interest. But we could take a number of objects and rank them in order of how warm they feel.

With ordinal data, one can use a variety of methods. For instance, one can compare the rank order of units on two different scales and calculate a rank-order correlation (Spearman's correlation; (Navarro, 2015, p. 146)). One can also find the median (middle) unit(s).

\subsubsection{Interval}

The next step up is interval level data. Here we can also order the units, but we can also measure the relative distance between the units. Furthermore, each point on the scale represents the same difference in the construct we are measuring. Thus, the difference between 3 and 4 is the same as that between 10 and 11. There is no true zero, however, so multiplication/division does not make sense to use. Note that this also includes percentages because they are based on division.

In the temperature example, Celsius is an example of an interval scale. The temperature difference between $10^{\circ} \mathrm{C}$ and $20^{\circ} \mathrm{C}$ is the same as that between $50^{\circ} \mathrm{C}$ and $60^{\circ} \mathrm{C}$. However, it does not make sense to say that $20^{\circ} \mathrm{C}$ is twice as warm as $10^{\circ} \mathrm{C}$. To see the nonsensicalness of it, consider the question of whether $10^{\circ} \mathrm{C}$ is $-100 \%$ as warm as $-10^{\circ} \mathrm{C}$.

\subsubsection{Ratio}

The final level of measurement is the ratio level. At this level we can do everything we could do earlier, but we also have a true zero which enables the use of multiplication/division.

In the temperature example, the Kelvin scale is a ratio scale. $0^{\circ} \mathrm{K}$ really is zero heat. $10^{\circ} \mathrm{K}$ really is twice as warm as $5^{\circ} \mathrm{K}$.

\subsubsection{Downgrading data}

Note that it is always possible to downgrade the level of data. For instance, if we began with ratio level data of temperature (say, in Kelvin), we could downgrade it to interval level data by standardizing the values (converting it to $\mathrm{Z}$ scores). This would set the mean value to 0 , but this 0 would not represent zero temperature and thus multiplication/division would stop making sense.

If we wanted to change ratio data to ordinal data, we could divide the datapoints into bins with unequal widths. This would make it the case that the distances between the bins were (in all likelihood) not equal but that they were still ordered. 
Finally, if we wanted to reduce the level to nominal, if we bin the datapoints at random or using some irrelevant feature. This would result in categories of data that were not in any particular order (unless by sheer chance).

\subsection{Typological data}

While some have typologists have proposed a more continuous approach to linguistic diversity (Daniel, 2011), many perhaps most typological variables are categorical. Figure 4 shows the distribution of the number of categories in each language feature in the WALS dataset.

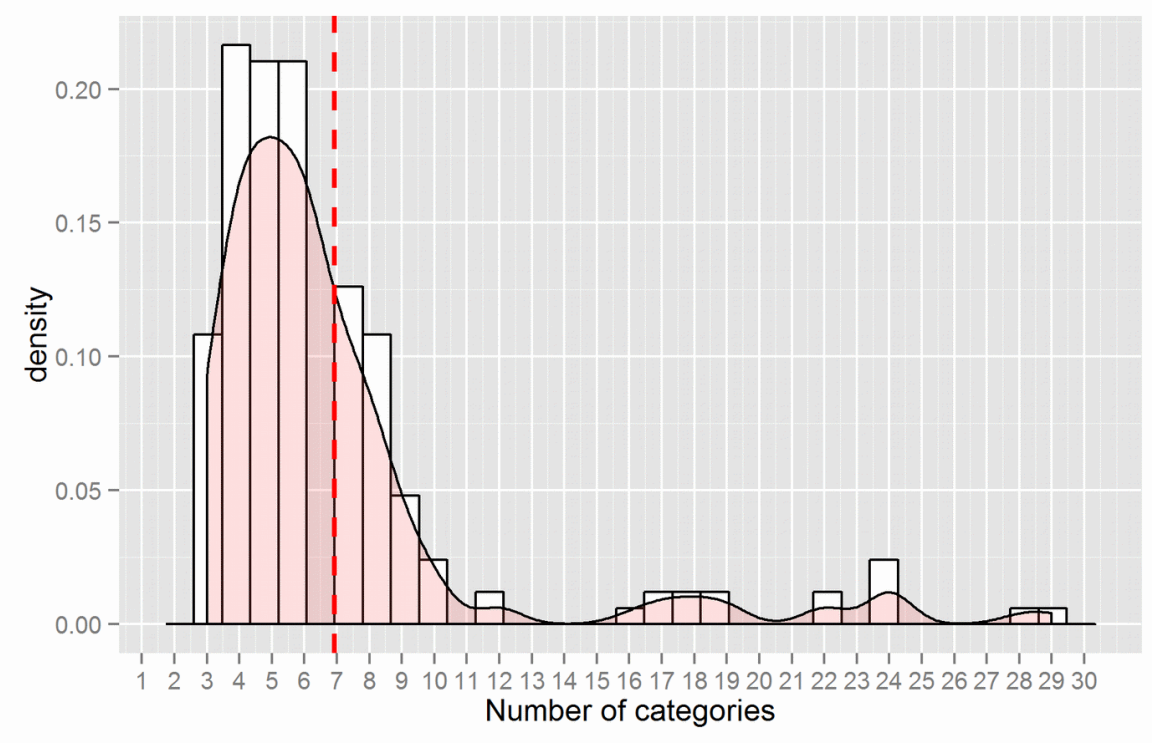

Figure 4: The distribution of number of categories by language feature in WALS. The red line is the mean.

The widespread use of categorical variables is different from (perhaps) most other areas of science, where the higher levels of data are more common. In psychology, for instance, probably the most common data level is interval or quasi-interval (Navarro, 2015, p. 18).

The use of categorical data seriously limits the statistical methods that can be used (Agresti, 2013), so it is important not to downgrade the level of data. Unfortunately, this seems to be common practice, at least in the WALS dataset. For instance, one language feature is 1A: Consonant Inventories (http://wals.info/chapter/1) which concerns the number of consonants found in the language. The data as given in WALS have 5 categories on an ordinal level: small, moderately small, medium, moderately large and large. Inspection of the definition of the these categories reveals that the distance between them are probably uneven (their ranges are given as: 6-14, 15-18, 19-25, 26-33, and $\geq 34$ ).

Unfortunately, it is apparently not possible to know because the data are only available in this form, not as the count data (ratio level) they were based on.

Some methods cannot be used on ordinal data, while others are biased when used on ordinal data. For instance, correlations (and other linear regression-type methods) have a downwards bias when the data have been put into bins (discretization). When the real correlation between two continuous variables is 0.5 , but we then put the one variable into 5 bins, the observed correlation falls to about 0.45 , which is about a $10 \%$ downward bias. If both variables were put into 5 bins, the bias would be about $15 \%$. If we 
forced the data into 2 bins for both variables, the observed correlation would be only 0.32, i.e. a downward bias of about 35\%. In practice this would mean that we would often fail to discover important relationships in the data (especially if using null significance testing methods, which are the norm (Cumming, 2012, 2014)).

To get a better understanding of the effects of discretization, use the interactive visualization available at http://emilkirkegaard.dk/understanding statistics/?app=discretization.

\section{Finding patterns in typological data}

As mentioned earlier, most proposed patterns have the form of conditional probabilities. From my reading of the typological literature, it seems that most of these were found manually, that is, a researcher manually looked up the relationship between two language features using a dataset like WALS.

However, it is possible to automatically find conditional probabilities in datasets by simply trying all the possible combinations using statistical computing. As before, I will be using data from WALS to showcase this.

\subsection{A note on probabilities}

A more sophisticated approach to conditional probabilities is the use of Bayes' rule (Bayesian statistics). This approach takes into account the overall or initial distribution of the data (prior probabilities) when calculating the resultant probabilities, then called posterior probabilities. This approach arguably produces better results. Due to space limitations, the details of this are not presented here, see e.g. Kruschke (2011) or online resources (such as http://betterexplained.com/articles/anintuitive-and-short-explanation-of-bayes-theorem/) for an introduction.

\subsection{Method}

To avoid complicated results, I restrict the number of predictor (independent) variables to 1 and use the same outcome (dependent) variable in all analyses. As the dependent variable, I used the first feature (1A Consonant Inventories).

Most statistical universals reported do not give the exact probabilities, but merely give a quantifier like most, suggesting that a probability $>0.50$ is sufficient. To avoid a very large number of results, I limited the results to those with posterior probabilities at or above 0.90 . Furthermore, to avoid statistical flukes of small samples, I limited the results to those that were based on at least 20 languages.

The WALS dataset has 192 features of which 1 was used as a the outcome. The remaining 191 were thus predictors. The outcome feature has 5 categories and the total number of categories across all 191 predictors is 1325 . Thus, a total of 7950 relationships were tested (less than $0.5 \%$ of possible combinations in the WALS dataset).

\subsection{Results}

Table 1 shows the tendencies that were found. 


\begin{tabular}{|c|c|c|c|c|c|}
\hline pattern \# & outcome category & pred var & pred category & postprob & $\mathbf{N}$ \\
\hline 1 & 1 Small & 6A Uvular Consonants & 1 None & 0.99 & 88 \\
\hline 2 & 2 Moderately small & 6A Uvular Consonants & 1 None & 0.92 & 112 \\
\hline 3 & 1 Small & 7A Glottalized Consonants & $\begin{array}{l}1 \text { No glottalized } \\
\text { consonants }\end{array}$ & 0.99 & 88 \\
\hline 4 & 2 Moderately small & 7A Glottalized Consonants & $\begin{array}{l}1 \text { No glottalized } \\
\text { consonants }\end{array}$ & 0.93 & 113 \\
\hline 5 & 1 Small & 11A Front Rounded Vowels & 1 None & 0.98 & 87 \\
\hline 6 & 2 Moderately small & 11A Front Rounded Vowels & 1 None & 0.97 & 117 \\
\hline 7 & 4 Moderately large & 11A Front Rounded Vowels & 1 None & 0.95 & 88 \\
\hline 8 & 5 Large & 11A Front Rounded Vowels & 1 None & 0.93 & 52 \\
\hline 9 & 3 Average & 18A Absence of Common Consonants & 1 All present & 0.93 & 187 \\
\hline 10 & 4 Moderately large & 18A Absence of Common Consonants & 1 All present & 0.97 & 91 \\
\hline 11 & 5 Large & 18A Absence of Common Consonants & 1 All present & 0.93 & 53 \\
\hline 12 & 1 Small & 19A Presence of Uncommon Consonants & 1 None & 0.94 & 84 \\
\hline 13 & 2 Moderately small & 19A Presence of Uncommon Consonants & 1 None & 0.92 & 112 \\
\hline 14 & 3 Average & 58A Obligatory Possessive Inflection & 2 Absent & 0.95 & 53 \\
\hline 15 & 1 Small & 73A The Optative & $\begin{array}{l}2 \text { Inflectional } \\
\text { optative absent }\end{array}$ & 1.00 & 27 \\
\hline 16 & 2 Moderately small & 73A The Optative & $\begin{array}{l}2 \text { Inflectional } \\
\text { optative absent }\end{array}$ & 0.92 & 45 \\
\hline 17 & 4 Moderately large & 108A Antipassive Constructions & 3 No antipassive & 0.93 & 25 \\
\hline 18 & 1 Small & 136A M T Pronouns & 1 No M-T pronouns & 0.92 & 24 \\
\hline 19 & 1 Small & 137A N M Pronouns & 1 No N-M pronouns & 0.92 & 24 \\
\hline 20 & 4 Moderately large & 137A N M Pronouns & 1 No N-M pronouns & 0.90 & 27 \\
\hline 21 & 3 Average & 90C Postnominal relative clauses & $\begin{array}{l}1 \text { Noun-Relative } \\
\text { clause (NRel) } \\
\text { dominant }\end{array}$ & 0.95 & 71 \\
\hline 22 & 1 Small & 144P NegSOV Order & 4 No NegSOV & 0.93 & 25 \\
\hline 23 & 2 Moderately small & 144P NegSOV Order & 4 No NegSOV & 1.00 & 28 \\
\hline 24 & 3 Average & 144P NegSOV Order & 4 No NegSOV & 0.92 & 45 \\
\hline 25 & 4 Moderately large & 144P NegSOV Order & 4 No NegSOV & 0.92 & 24 \\
\hline 26 & 4 Moderately large & 144Q SNegOV Order & 4 No SNegOV & 0.92 & 24 \\
\hline 27 & 2 Moderately small & 144H NegSVO Order & 4 No NegSVO & 0.96 & 22 \\
\hline 28 & 3 Average & 144H NegSVO Order & 4 No NegSVO & 0.96 & 46 \\
\hline 29 & 4 Moderately large & 144H NegSVO Order & 4 No NegSVO & 0.93 & 26 \\
\hline 30 & 1 Small & 143G Minor morphological means of signaling negation & 4 None & 1.00 & 58 \\
\hline 31 & 2 Moderately small & 143G Minor morphological means of signaling negation & 4 None & 1.00 & 89 \\
\hline
\end{tabular}




\begin{tabular}{|c|c|c|c|c|c|}
\hline 32 & 3 Average & 143G Minor morphological means of signaling negation & 4 None & 0.99 & 142 \\
\hline 33 & 4 Moderately large & 143G Minor morphological means of signaling negation & 4 None & 0.97 & 70 \\
\hline 34 & 5 Large & 143G Minor morphological means of signaling negation & 4 None & 1.00 & 37 \\
\hline 35 & 1 Small & 25B Zero Marking of A and P Arguments & 2 Non-zero marking & 0.96 & 25 \\
\hline 36 & 2 Moderately small & 25B Zero Marking of A and P Arguments & 2 Non-zero marking & 1.00 & 39 \\
\hline 37 & 3 Average & 25B Zero Marking of A and P Arguments & 2 Non-zero marking & 0.90 & 47 \\
\hline 38 & 4 Moderately large & 25B Zero Marking of A and P Arguments & 2 Non-zero marking & 0.97 & 31 \\
\hline 39 & 4 Moderately large & 108B Productivity of the Antipassive Construction & 4 no antipassive & 0.93 & 25 \\
\hline 40 & 1 Small & 58B Number of Possessive Nouns & 1 None reported & 0.94 & 30 \\
\hline 41 & 2 Moderately small & 58B Number of Possessive Nouns & 1 None reported & 0.95 & 37 \\
\hline 42 & 3 Average & 58B Number of Possessive Nouns & 1 None reported & 0.95 & 53 \\
\hline 43 & 4 Moderately large & 58B Number of Possessive Nouns & 1 None reported & 1.00 & 36 \\
\hline 44 & 1 Small & 79B Suppletion in Imperatives and Hortatives & $\begin{array}{l}5 \text { None (= no } \\
\text { suppletive } \\
\text { imperatives reported } \\
\text { in the reference } \\
\text { material) }\end{array}$ & 1.00 & 25 \\
\hline
\end{tabular}

Table 1: Predictive patterns found for Consonant Inventory (1A) in the WALS dataset.

As can be seen, of the about 8000 patterns tested, only 44 were found to meet the fairly strict inclusion criteria, which is about $0.6 \%$ of those tested.

Note that the above does not include probabilities that are strong, but in the negative direction (i.e. close to 0 instead of 1 ). In other words, the results do not include patterns of the type:

5. If language L has feature A, then it is unlikely (posterior probability) that it has feature B.

Such patterns may also be of interest, but would be partial duplicates of the near-1 probability patterns reported in the table. In my reading of the typological literature, I have not come across any proposed negative patterns.

\subsection{Are there really patterns in language features?}

How do we make sense of results like this? Due to the massive number of combinations tested, many of the above results will probably be statistical flukes (false positives) (Ioannidis, 2005; Ioannidis, Tarone, \& McLaughlin, 2011). While one cannot (easily) be certain that a given pattern is not a fluke², one can examine whether the overall distribution of patterns looks like one obtained from simulated data without any pre-specified patterns.

To do this, I simulated data using the summary statistics from the actual WALS dataset. That is, I simulated a dataset with the same number of languages, same number of features and the features had the same distributions (number of categories, percentages of languages having each feature), but without any pre-specified patterns between the features. I did not attempt to simulate missing data since

2 One could adjust for multiple testing á la Bonferroni or similar, but it is outside the scope of this paper (Navarro, 2015, p. 445). 
this would be difficult.

After this, I calculated all the posterior probabilities with samples $\geq 20$ languages in both the real WALS dataset and the simulated version. If there really are no patterns in the WALS dataset, the distributions of the posterior probabilities should look similar for the real and the simulated data. Figures 5 and 6 show the distributions.

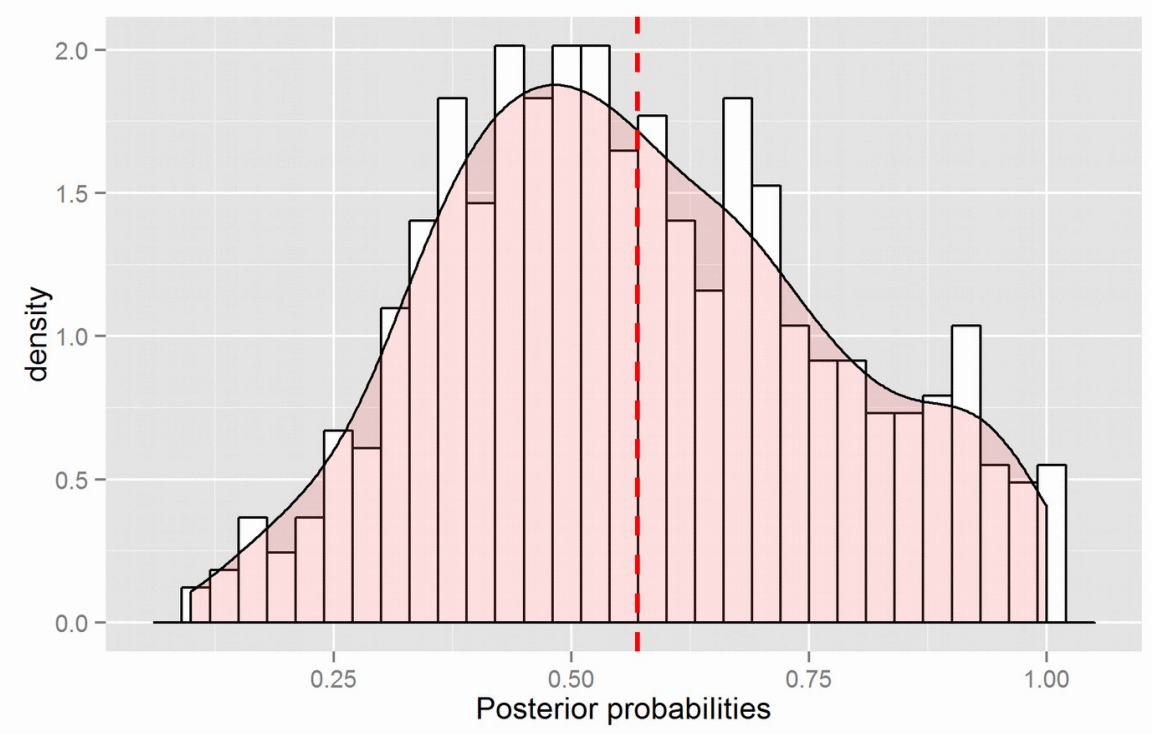

Figure 5: All posterior probabilities based on at least 20 languages. WALS data. The red line is the mean.

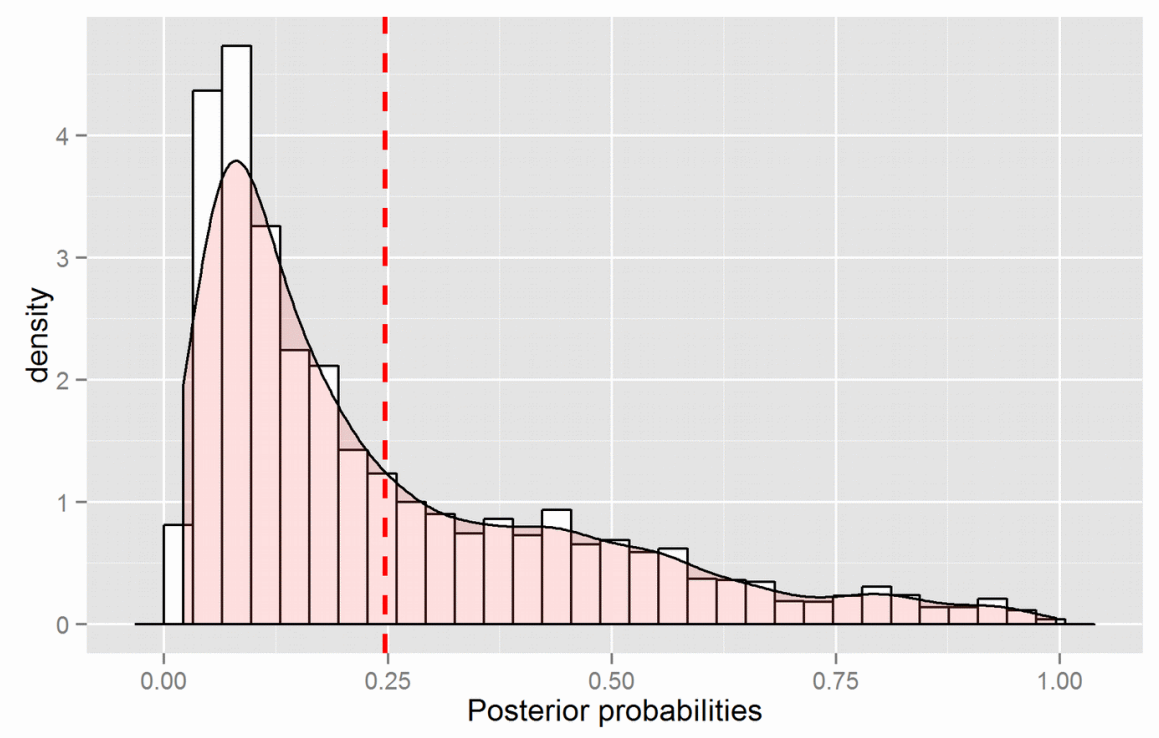

Figure 6: All posterior probabilities based on at least 20 languages.

Simulated WALS-like data with no patterns. The red line is the mean.

We see a clear difference. The simulated scores cluster around the low probabilities because this reflects the approximately evenly distributed posterior probabilities (i.e. if a feature has 7 categories, 
each would have $1 / 7=14 \%$ chance of being chosen. Recall that the mean number of features is about 7 , cf. Figure 4). The WALS data posteriors, however, cluster around 0.50 reflecting that there really are probabilistic relationships between many of the language features.

\section{Spatial autocorrelation}

The very unchancelike results found in Section 4 could however reflect confounds. In particularly, it has been noted that areal and family relatedness effects may result in patterns without there being some deeper typological relationship (Bickel, 2011; Maslova, 2000; Moravcsik, 2013). Effects like these have been studied in other areas of science and even have a name: spatial autocorrelation. Positive autocorrelation means that units that are closer to each other in some space (e.g. in time, in physical space or in family space) are more similar. When spatial autocorrelation is present in datasets, conclusions derived using methods not designed to take the phenomenon into account may give misleading results: either because we seem to find evidence of an effect that isn't real (false positive) or fail to find evidence of a real cause due to noise from other effects (false negative) (Kirkegaard, 2015; Radil, 2011).

Since languages are spoken by people and people live somewhere, languages effectively have a (physical) location. Because languages are often intimately related to sovereign states, the location of a given language may be peculiar; it can even be split into multiple areas. For instance, Spanish is spoken (as a first language) in many parts of the world including the Americas, Europe and Africa and South Asia ("Hispanophone," 2016). In WALS, a single longitude-latitude pair is given for the languages which may be misleading. In the case of Spanish, this location is just south of Madrid, Spain (coordinates: -4, 40), despite most Spanish speakers living in the Americas.

Analyzing spatial data is a complex task, but statistical methods for doing so have been emerging the last couple of decades and especially the last decade (Armstrong et al., 2014; Bivand, Pebesma, \& Gómez-Rubio, 2013; Dube, 2014; Plant, 2012). Spatial statistics has also been used within the field of linguistics such as with the analysis of dialect maps (Lee \& Kretzschmar, 1993; Rumpf, Pickl, Elspass, König, \& Schmidt, 2009), but it does not seem to be common practice.

\subsection{Spatial autocorrelation in language features}

Bickel (2011) gives an example of a spatially autocorrelated feature (NP order) shown in Figure 7. One can easily see that languages that are spatially near each other tend to have similar a NP order. There are multiple standard methods that can be used to quantify the strength of the spatial autocorrelation. The most commonly used is Moran's I (Kirkegaard, 2015; Radil, 2011). However, because Moran's I is based on correlations (sort of) which require at the very least ordinal level data, it cannot be used on many language features.

For the purpose of examining spatial autocorrelation in population genetical data, I developed a method called k-nearest spatial neighbor regression (KNSNR) (Kirkegaard, 2015). The method consists of the following steps:

1. Calculate the spatial distance between each pair of cases in the chosen space.

2. For each case: 
a) Find that case's k nearest neighbors.

b) Use the values of the neighbors' outcomes to estimate the value of the outcome in the case of interest and save the estimate(s).

This produces one or more estimated values for each case in the dataset. I worked with numeric values when I developed the method and used the mean value as the estimated value. However, this cannot be done with categorical data. Instead, one might use the most common value among the neighbors as the best guess (the mode). If there is a tie, then one can use all the tied values.

The estimated values can then be compared with the actual values of the cases in the dataset to see how well these could be estimated from the neighboring cases. In the original version of KNSNR I used the (Pearson) correlation as the measure of the relationship between the estimated and actual values.

Because this cannot be used for categorical values, some other method must be used. I decided to use a simple method, namely that if the estimated value is correct, a score of 1 is given, and if it is not, a score of 0 is given. If there is more than one estimated value, the score is divided by the number of such values. For instance, if there were two estimated values and the true value was among them, the score is $1 / 2=0.5$. Of course, if the true value was not among them, the score would be 0 no matter how many estimated values there were ( 0 divided by any integer is 0 ). When the estimates have been scored, one can calculate an overall measure of the values by taking the mean. However, before doing that, there are two complications. 


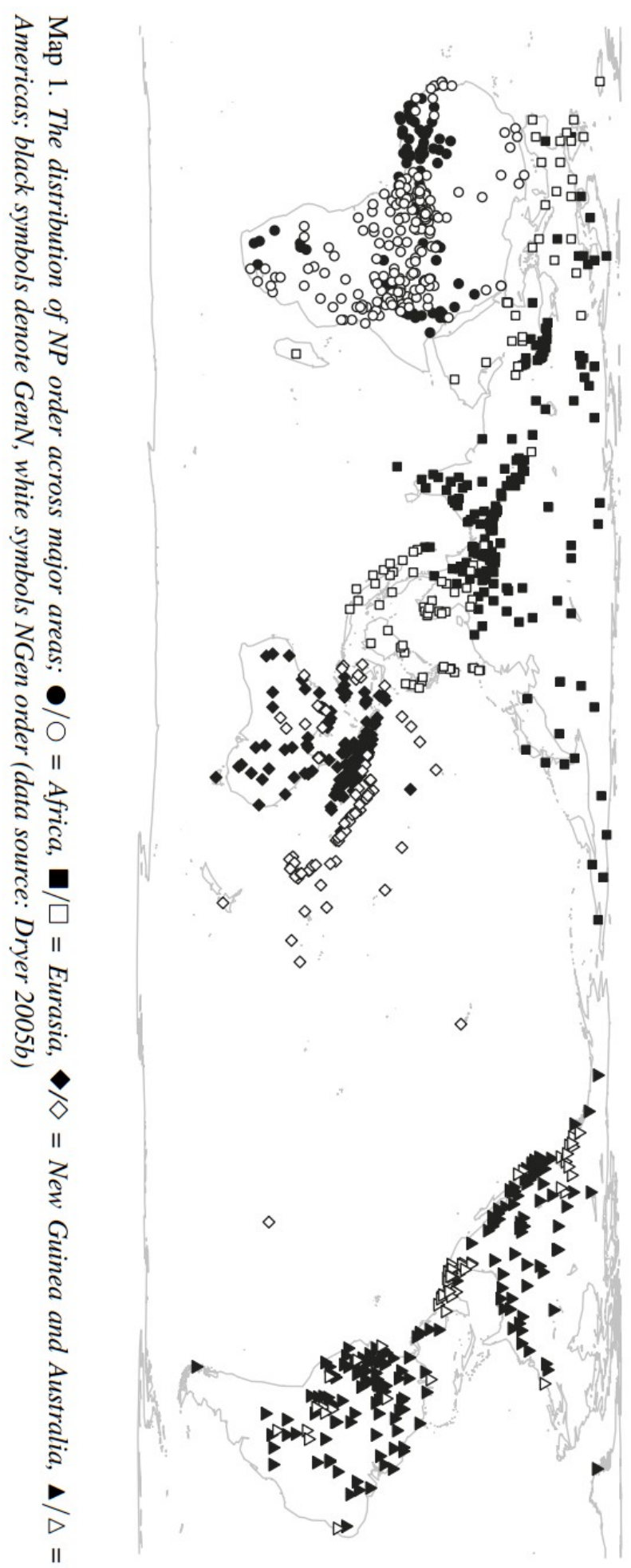

Figure 7: Spatial distribution of NP order. Languages that are near each other are similar in their NP order (spatial autocorrelation). From Bickel (2011). 


\subsection{Choosing a value of $k$}

The first complication is that one must choose a value for $\mathrm{k}$. Recall, that this controls the number of neighbors used in the prediction. What this does is choose the size of the neighborhood taken into account, or the 'zoom-level'. Analyses of simulated (numeric) data showed that using $\mathrm{k}=3$ yielded the best results (Kirkegaard, 2015). Analyses of real data in a large study yielded a best k of 2 or 3 (Fuerst \& Kirkegaard, 2016). However, the same value of $\mathrm{k}$ may not be optimal for categorical data or for linguistic data in general. To determine what might be a good value for $\mathrm{k}$, I calculated the spatial autocorrelation mean score for each value of $\mathrm{k}$ between 1 and 20 for the first 5 variables in WALS. Figure 8 shows the results.

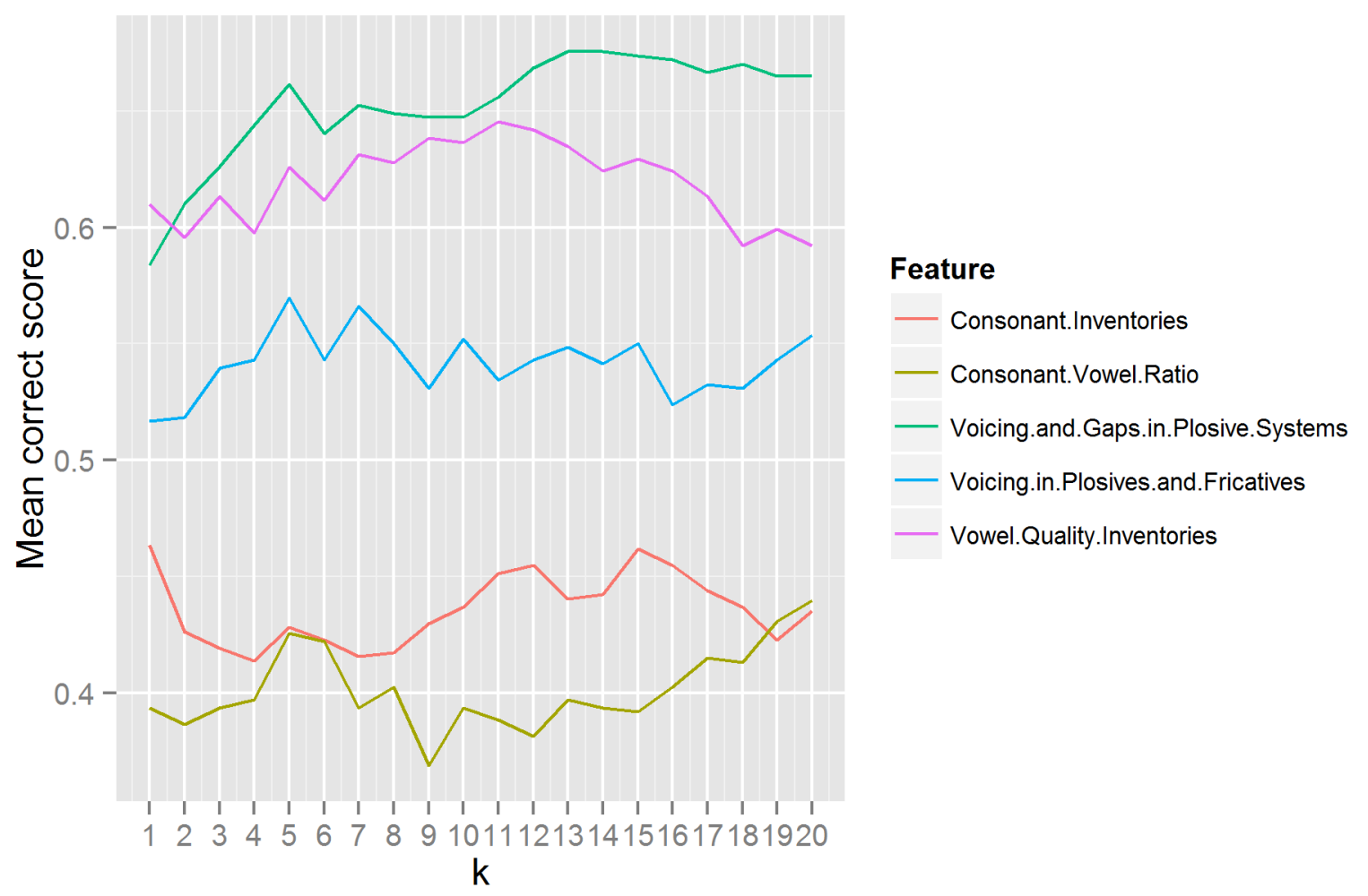

Figure 8: Mean correct score across values of $k$ for 5 language features.

The best results were found with k=5, but closely followed by k=15 as shown in Figure 9 . 


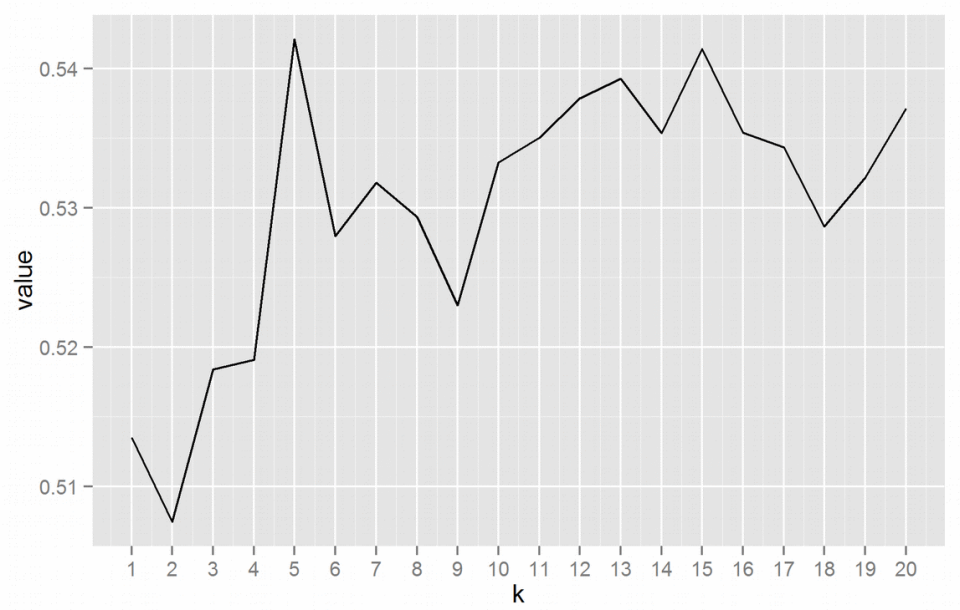

Figure 9: Mean correct by across values of $k$.

Because the $\mathrm{k}=5$ result looked like a coincidence, $\mathrm{I}$ used $\mathrm{k}=15$ for the main analysis.

\subsection{The base rate of correct guesses}

The secondary complication is that while we have estimated the features of the languages from their neighbors, it is possible that we could have done just as well using non-neighbors. That is, if a given feature is the most common across all languages, we could use that as our estimate for every language and thus get most languages right without taking into account their neighbors at all. We can refer to the mean score obtained from just guessing from all languages the base rate of correct guesses. If there is spatial autocorrelation in the data, we should be able to do better than this by looking at languages' neighbors. Thus, we can calculate the gain in the mean score from estimating from the languages' neighbors versus just using the estimate from all languages.

\subsection{Results}

Figures 10, 11 and 12 show the distributions of mean scores using the languages' k (15) nearest neighbors, using all languages (base rate) and the gain in mean scores above the base rate of correct guesses.

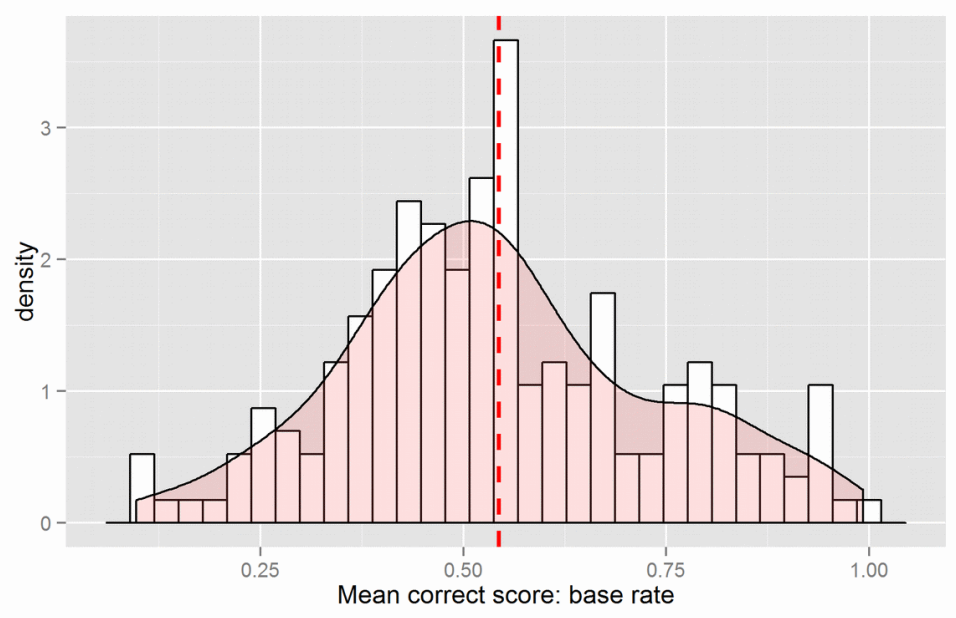

Figure 10: Distribution of mean scores of the base rate estimates. 


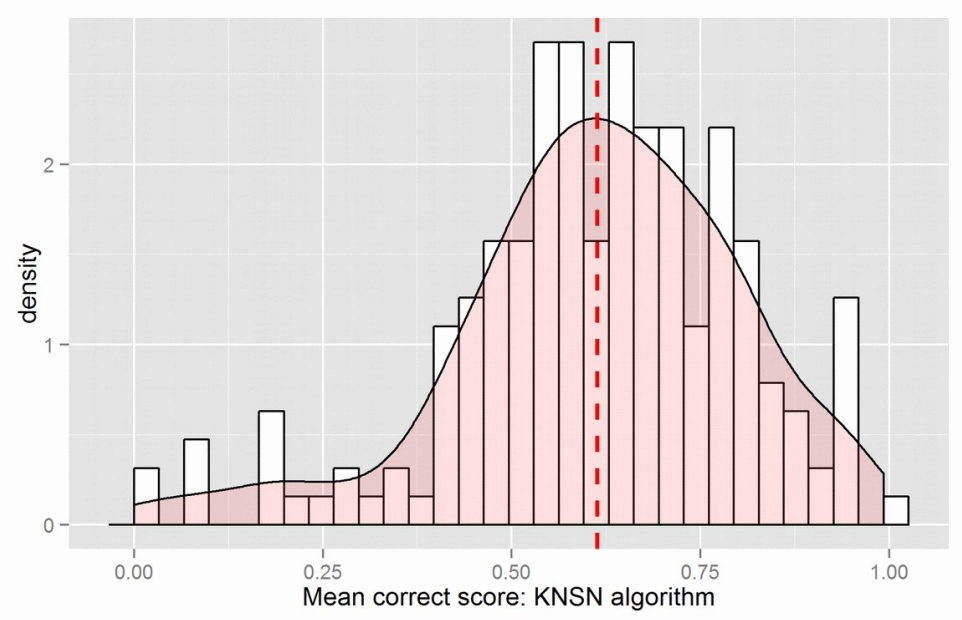

Figure 11: Distribution of mean scores of the k-nearest neighbor estimates.

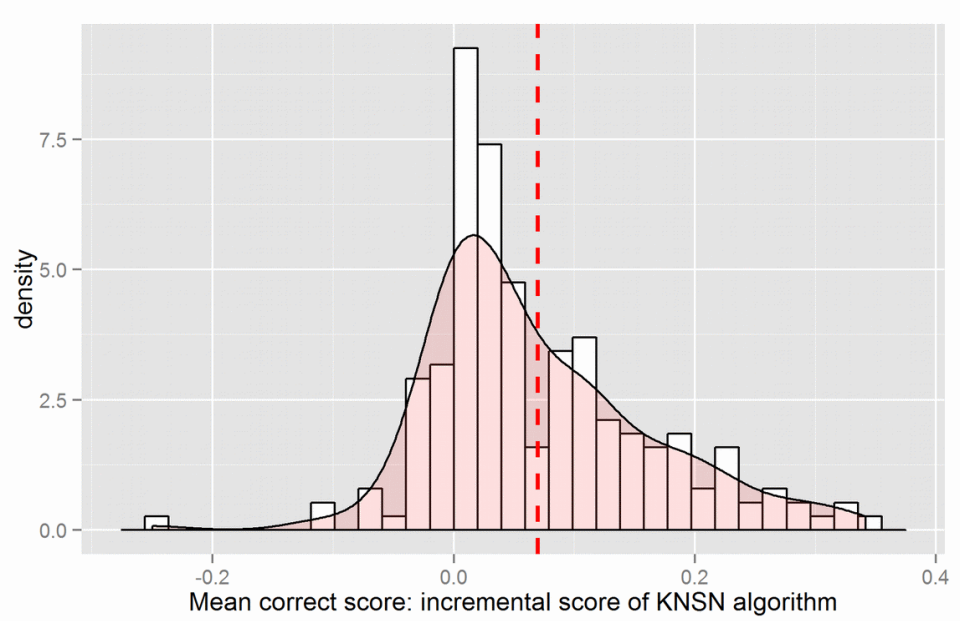

Figure 12: Distribution of mean scores of the gain in scores using the KNSN algorithm versus the base rate.

We see that using the neighbors' values did improve the mean score by about 0.07 . In other words, if one looks at languages' neighbor' languages one can predict languages features somewhat better than one can from just considering the base rate. This means that the data shows some degree of spatial autocorrelation, which must be taken into account. It does not necessarily reflect areal effects, but could also reflect that languages tend to be located near familiarly related languages, which are similar in the language features.

\section{Discussion and conclusion}

This paper presented three major findings. The first (Section 2.1) is that missing data does not seem to be tied to macroareas ('continents'). This is welcome news because missing data that isn't missing completely at random can create serious bias in the results (Donders, van der Heijden, Stijnen, \& Moons, 2006). 
The second (Section 4) is that one can identify typological patterns automatically using statistical computing. This was showcased by applying the method to one language feature, the size of the consonant inventory, and reporting 44 patterns related to that feature all of which had posterior probabilities above 0.90 and a sample size of at least 20 (Table 1).

The third (Section 4.4) is that an analysis of all the posterior probabilities calculated from one language feature in the WALS dataset with samples sizes of at least 20 were markedly differently distributed compared to simulated data highly similar to the WALS data.

The four (Section 5) is that the language features in WALS show some degree of spatial autocorrelation. This means that results from a method that does not take this into account cannot be taken as face value as it may reflect a spatially autocorrelated confound.

The analyses presented in this paper show that typology can benefit from statistical methodology, in particular statistical computing and spatial statistics. Fortunately, there seems to be an increased interest in statistical approaches to typology recently (Bickel, 2011; Dunn, Greenhill, Levinson, \& Gray, 2011; Maslova, 2000; Piantadosi \& Gibson, 2014), but it is not (yet) widespread.

\section{Supplementary material}

Supplementary materials including complete datasets, R code, high quality figures and outputted datasets are available at the Open Science Framework repository: https://osf.io/d7tzf/.

\section{References}

Agresti, A. (2013). Categorical data analysis (3rd ed). Hoboken, NJ: Wiley.

Armstrong, D. A., Bakker, R., Carroll, R., Hare, C., Poole, K. T., \& Rosenthal, H. (2014). Analyzing spatial models of choice and judgment with R. Hoboken: Taylor and Francis. Retrieved from http://www.crcnetbase.com/doi/book/10.1201/b16486

Bickel, B. (2011). Statistical modeling of language universals. Linguistic Typology, 15(2), 401-413.

Bivand, R. S., Pebesma, E., \& Gómez-Rubio, V. (2013). Applied Spatial Data Analysis with R. New York, NY: Springer New York. Retrieved from http://link.springer.com/10.1007/978-1-46147618-4

Brown, C. H. (1976). general principles of human anatomical partonomy and speculations on the growth of partonomic nomenclature1. American Ethnologist, 3(3), 400-424. http://doi.org/10.1525/ae.1976.3.3.02a00020

Chalmers, A. F. (1999). What is this thing called science? (3rd ed). Indianapolis: Hackett Pub. Cox, D. R. (2006). Principles of statistical inference. Cambridge; New York: Cambridge University 
Press. Retrieved from http://public.eblib.com/choice/publicfullrecord.aspx?p=321138

Cumming, G. (2012). Understanding the new statistics: effect sizes, confidence intervals, and metaanalysis. New York: Routledge.

Cumming, G. (2014). The New Statistics Why and How. Psychological Science, 25(1), 7-29. http://doi.org/10.1177/0956797613504966

Daniel, M. (2011). Linguistic Typology and the Study of Language. In The Oxford Handbook of Linguistic Typology. Oxford University Press. Retrieved from http://www.oxfordhandbooks.com/view/10.1093/oxfordhb/9780199281251.001.0001/oxfordhb9780199281251-e-004

Donders, A. R. T., van der Heijden, G. J. M. G., Stijnen, T., \& Moons, K. G. M. (2006). Review: A gentle introduction to imputation of missing values. Journal of Clinical Epidemiology, 59(10), 1087-1091. http://doi.org/10.1016/j.jclinepi.2006.01.014

Dryer, M. S., \& Haspelmath, M. (Eds.). (2013). WALS Online. Leipzig: Max Planck Institute for Evolutionary Anthropology. Retrieved from http://wals.info/

Dube, J. (2014). Spatial econometrics using microdata. Hoboken, NJ: ISTE Ltd/John Wiley and Sons Inc.

Dunn, M., Greenhill, S. J., Levinson, S. C., \& Gray, R. D. (2011). Evolved structure of language shows lineage-specific trends in word-order universals. Nature, 473(7345), 79-82. http://doi.org/10.1038/nature09923

Fuerst, J., \& Kirkegaard, E. O. W. (2016). Admixture in the Americas: Regional and national differences. Mankind Quarterly.

Greenberg, J. H. (Ed.). (1966). Universals of language: report of a conference held at Dobbs Ferry, New York, April 13-15, 1961 (2. ed). Cambridge, Mass.: M.I.T. Press.

Grenoble, L. A. (2011). Language ecology and endangerment. In P. Austin \& J. Sallabank (Eds.), The Cambridge Handbook of Endangered Languages. Cambridge University Press. Retrieved from http://dx.doi.org/10.1017/CBO9780511975981.002

Hispanophone. (2016, January 3). In Wikipedia, the free encyclopedia. Retrieved from https://en.wikipedia.org/w/index.php?title=Hispanophone\&oldid=698090169 
Ioannidis, J. P. A. (2005). Why Most Published Research Findings Are False. PLoS Med, 2(8), e124. http://doi.org/10.1371/journal.pmed.0020124

Ioannidis, J. P. A., Tarone, R., \& McLaughlin, J. K. (2011). The False-positive to False-negative Ratio in Epidemiologic Studies: Epidemiology, 22(4), 450-456. http://doi.org/10.1097/EDE.0b013e31821b506e

Kirkegaard, E. O. W. (2015). Some methods for measuring and correcting for spatial autocorrelation. The Winnower. Retrieved from https://thewinnower.com/papers/2847-some-methods-formeasuring-and-correcting-for-spatial-autocorrelation

Kruschke, J. K. (2011). Doing bayesian data analysis: a tutorial with R and BUGS. Burlington, MA: Academic Press.

Lee, J., \& Kretzschmar, W. A. (1993). Spatial analysis of linguistic data with GIS functions. International Journal of Geographical Information Systems, 7(6), 541-560. http://doi.org/10.1080/02693799308901981

LePore, E., \& Cumming, S. (2009). Meaning and argument: an introduction to logic through language (2nd ed). Chichester, U.K. ; Malden, MA: Wiley-Blackwell.

Libert, A. R. (2013a). What can Pragmaticists Learn from Studying Artificial Languages? In A. Capone, F. Lo Piparo, \& M. Carapezza (Eds.), Perspectives on Linguistic Pragmatics (Vol. 2, pp. 397-432). Cham: Springer International Publishing. Retrieved from http://link.springer.com/10.1007/978-3-319-01014-4_16

Libert, A. R. (2013b, May 29). Theoretical linguistics and artificial languages. Retrieved from http:/hiphilangsci.net/2013/05/29/theoretical-linguistics-and-artificial-languages/

Maslova, E. (2000). A dynamic approach to the verification of distributional universals. Linguistic Typology, 4(3). http://doi.org/10.1515/lity.2000.4.3.307

Moravcsik, E. A. (2011). Explaining Language Universals. In J. J. Song (Ed.), The Oxford Handbook of Linguistic Typology. Retrieved from http://www.oxfordhandbooks.com/view/10.1093/oxfordhb/9780199281251.001.0001/oxfordhb9780199281251-e-005

Moravcsik, E. A. (2013). Introducing Language Typology. Cambridge: Cambridge University Press. 
Navarro, D. (2015). Learning Statistics with R. Retrieved from http://health.adelaide.edu.au/psychology/ccs/teaching/lsr/

Okrent, A. (2009). In the land of invented languages: Esperanto rock stars, Klingon poets, Loglan lovers, and the mad dreamers who tried to build a perfect language (1st ed). New York: Spiegel \& Grau.

Piantadosi, S. T., \& Gibson, E. (2014). Quantitative Standards for Absolute Linguistic Universals. Cognitive Science, 38(4), 736-756. http://doi.org/10.1111/cogs.12088

Plant, R. E. (2012). Spatial data analysis in ecology and agriculture using R. Boca Raton: CRC Press. Retrieved from http://www.crcnetbase.com/isbn/9781439819142

Radil, S. M. (2011). Spatializing social networks: making space for theory in spatial analysis. University of Illinois at Urbana-Champaign. Retrieved from https://www.ideals.illinois.edu/handle/2142/26222

Ramat, P. (2011). The (early) history of linguistic typology. In The Oxford Handbook of Linguistic Typology. Oxford University Press.

Rumpf, J., Pickl, S., Elspass, S., König, W., \& Schmidt, V. (2009). Structural analysis of dialect maps using methods from spatial statistics. Zeitschrift Für Dialektologie Und Linguistik, 76(3), 280308.

Sokal, A. (2008). Beyond the hoax: Science, philosophy and culture. Oxford University Press. Retrieved from https://www.google.com/books? hl=en\&lr=\&id=SjBdUTpf184C\&oi=fnd\&pg=PT2\&dq=alan+sokal+alan+sokal+beyond\&ots=y EVp2pTJCR\&sig=zxG0M3uL7wCsx2K46hOJK15eFts

Velupillai, V. (2012). An introduction to linguistic typology. Amsterdam ; Philadelphia: John Benjamins Pub. Co. 